Case report

\title{
Trans-arterial and trans-venous interventional radiology for an elderly patient with life-threatening pelvic injury after accidental falling due to life-threatening cardiac arrhythmia: a case report Junya Morozumi*, Takao Arai and Shöichi Ohta
}

Address: Department of Emergency and Critical Care Medicine, Tokyo Medical University Hospital Hachioji Medical Center, Tokyo, 193-0998, Japan

Email: JM* - junya99@tokyo-med.ac.jp; TA - takaoarai21@hotmail.com; SO - sho-ohta@tokyo-med.ac.jp

* Corresponding author

Received: 27 February 2009 Accepted: 18 June 2009 Published: 20 July 2009

Cases Journal 2009, 2:6222 doi: 10.4076/1757-1626-2-6222

This article is available from: http://casesjournal.com/casesjournal/article/view/6222

(c) 2009 Morozumi et al; licensee Cases Network Ltd.

This is an Open Access article distributed under the terms of the Creative Commons Attribution License (http://creativecommons.org/licenses/by/3.0), which permits unrestricted use, distribution, and reproduction in any medium, provided the original work is properly cited.

\begin{abstract}
Introduction: A 79-year man, admitted to our emergency department after accidental falling with syncope, had pelvic fractures and complete atrioventricular (AV) block.

Case presentation: He received transvenous pacer placement for complete AV block and required hemodynamic stability. His heart rate was successfully controlled. However, secondary deterioration of his hemodynamics concerning of pelvic fractures was occurred and immediate trancatheter arterial embolization was performed. Final angiography showed no findings of bleeding and he was discharged intensive care unit in good condition.

Conclusion: Combined transarterial and transvenous interventional radiology is an effective and safety resuscitation technique for an elderly with secondary life-threatening injury after accidental falling due to life-threatening cardiac arrhythmia.
\end{abstract}

\section{Introduction}

Syncope is a clinical symptom which is characterized by a transient loss of consciousness, usually leading to accidental falling about one third of the population aged 65 or more $[1,2]$. Of $5-10 \%$ of the population result in secondary severe injuries after accidental falling, which is recognized as one of the lethal complications of transient loss of consciousness $[3,4]$. In the elderly, the number of patients who fall due to syncope has also increased $[5,6]$ and more than $90 \%$ of hip fractures are the result of a fall [7].
In the present study, we treated an elderly patient with a life-threatening pelvic injury after accidental falling secondary to syncope due to complete atrioventricular (AV) block using interventional radiology (IVR).

\section{Case presentation}

A 79-year-old Asian man was admitted to our emergency department (ED) after accidental falling with syncope from 2nd floor to the ground at his house. On examination, he had an E3V3M5 Glasgow Coma Scale score with restlessness, a presenting blood pressure of 
$104 / 55 \mathrm{mmHg}$, heart rate of 35 beats/minute, and a respiratory rate of $24 /$ minute on admission. He immediately received initial trauma resuscitation on the basis of the Advanced Trauma Life Support guidelines [8]. Initial electrocardiogram showed complete AV block in the ED (Figure 1). Though he underwent administration of $0.5 \mathrm{mg}$ of atropine and transcutaneous pacing immediately in primary care, his heart rate showed no improvement. Focused assessment with sonography for trauma and chest X-ray in the ED were interpreted as normal. Pelvic X-ray showed pelvic fractures in the sacrum, right pubic rami, and the ileum. Contrast-enhanced computed tomography (CT) scan of the chest, abdomen and pelvis showed hematoma on the right side of the pelvic cavity that compressed the bladder in the obturator internus, the iliacus and in the gluteus medius muscle of the fracture area and extrapelvic hematoma with contrast medium extravasation (Figure 2). The dislocation of the right elbow was also determined. The injury severity score, which is based on the abbreviated injury scale (AIS) severity values and derives from the sum of the squares of the highest AIS values from each of the 3 most severely injured body regions, was 20 .

Despite administration of initial fluid resuscitation using Ringer's lactate, his systolic blood pressure (SBP) deteriorated to $70 \mathrm{mmHg}$ and heart rate of 30 beats/minutes. We were concerned about cardiogenic shock or hemorrhagic shock, and therefore decided to place a transvenous pacer for complete $\mathrm{AV}$ block and to perform transcatheter arterial embolization (TAE) for the pelvic injury with massive fluid resuscitation in primary trauma resuscitation. We initially transferred him to the angiography room and initiated IVR to place the transvenous pacer because we found no improvement of his heart rate in spite of administration of atropine and placement of a transcutaneous pacemaker in the ED. The transvenous pacer was successfully placed for 1 hour, and his SBP had recovered to $100 \mathrm{mmHg}$. After completion of placement of the transvenous pacer, his heart rate was controlled at 60 beats/minute. However, his hemodynamic status soon deteriorated to a SBP of $79 \mathrm{mmHg}$. We continuously initiated TAE for secondary deterioration of his hemodynamic status concerning of pelvic injury. Initial pelvic

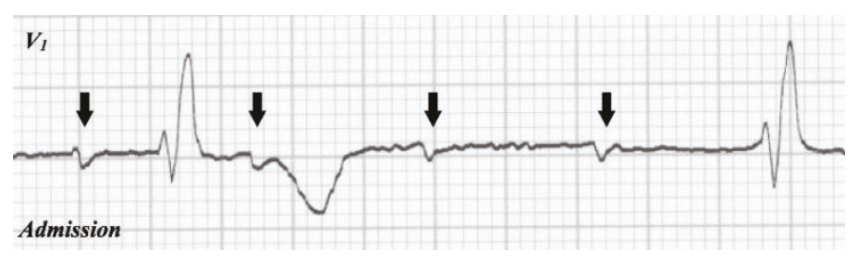

Figure I. Electrocardiogram of the patient showing complete atrioventricular block. P wave (black arrow).

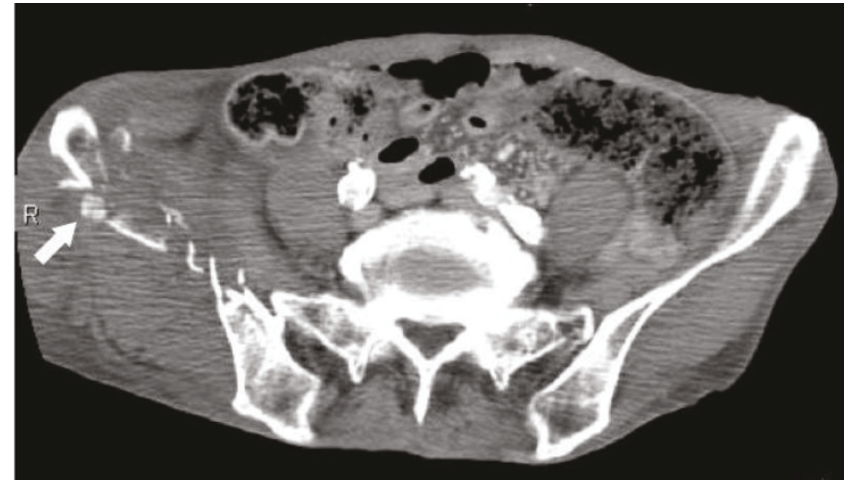

Figure 2. Computed tomography showed pelvic fractures with contrast medium extravasation (white arrow).

angiography showed multiple contrast medium extravasation from the branches of the right internal iliac artery (Figure 3). On completion of TAE, using stainless coils and gelatin sponge particles for the pelvic fractures, angiography showed no findings of bleeding (Figure 4). The patient required hemodynamic stability with a SBP of $150 \mathrm{mmHg}$ and heart rate was controlled at 60 beat/ minutes. After completion of TAE, he was admitted to our intensive care unit (ICU). At 12 hours after admission, he received a transfusion of 5 units of red blood cells and 10 units of fresh frozen plasma and his hemodynamic status remained stable. He was discharged ICU on hospital day 2, and his blood culture from admission to 24 hours after admission was detailed in Table 1.

\section{Discussion}

This patient had potentially fatal complications after accidental falling due to complete AV block, suffering severe pelvic injury, resulting in secondary hemorrhagic shock. Syncope is a clinical symptom, which is characterized by a transient, and self-limited loss of consciousness

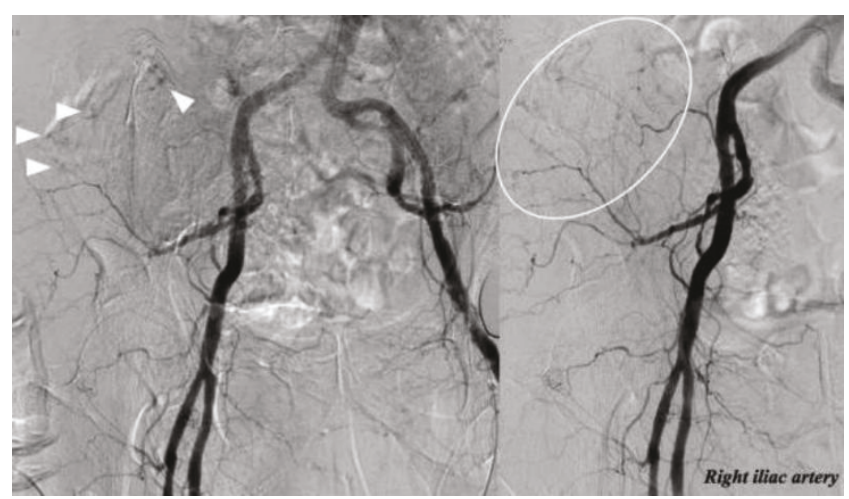

Figure 3. Pelvic angiography showed multiple contrast medium extravasation (white arrow and white circle). 


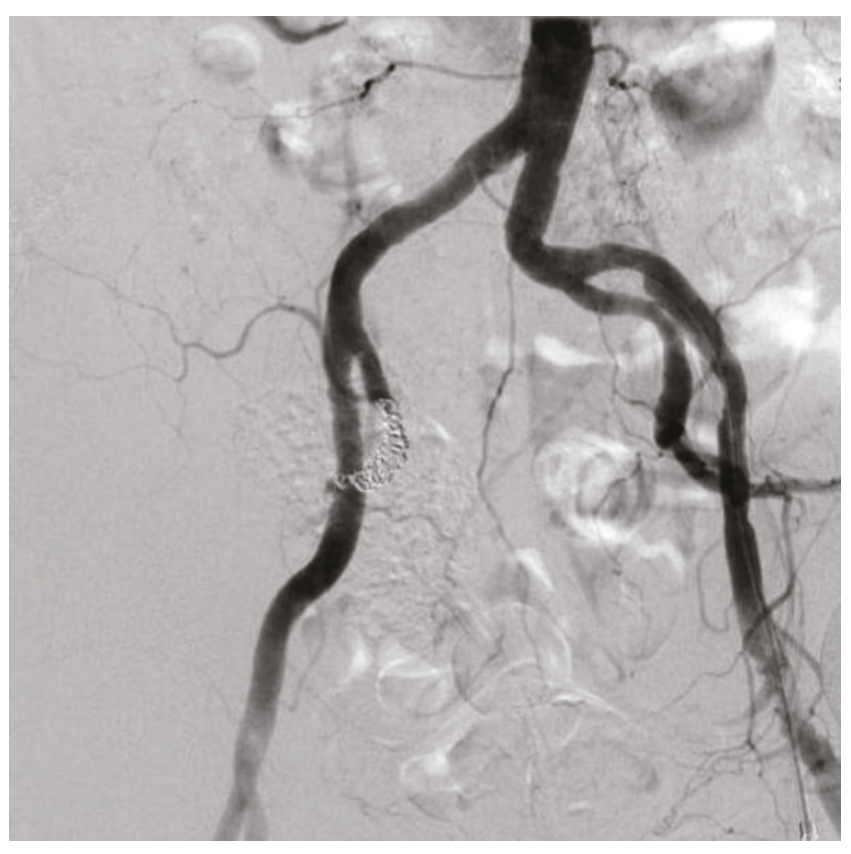

Figure 4. After completion of transcatheter arterial embolization, the contrast medium extravasation halted.

due to temporary reduction of cerebral perfusion [2]. For the elderly, syncope is a common problem in the health care setting, and attacks account for at least $6 \%$ of hospital visits [9]. Moreover, syncope usually leads to a secondary accidental fall, and the risk of accidental falling has been found to be greater than $20 \%$ per year for the elderly over 65 years [10]. In most cases, the syncopal condition is benign but in a minority, the condition can be sometimes life-threatening. Bartoletti et al. reported that 33\% of the prevalence of secondary trauma among the elderly patients with syncope is due to cardiac arrhythmias [11]. According to the European Society of Cardiology Guidelines on Syncope, complete AV block was one of the lifethreatening cardiac arrhythmia causes of syncope and the most common reason for pacemaker implantation [12]. In the present case, he soon received administration of atropine and placement of a transcutaneous pacemaker for complete AV block on the basis of the 2005 American Heart Association guidelines [13]. However, no hemodynamic improvement was recognized. Thus, we decided to place a transvenous pacer using IVR instead of surgical pacemaker implantation due to the hemodynamic instability in this elderly patient.

However, a hematoma with contrast medium extravasation by pelvic fractures was detected on CT scan, which meant a life-threatening situation requiring surgical or radiological intervention. Control of the arterial and venous hemorrhage, and prevention of exacerbation of his hemodynamic status and coagulation state was deemed essential. For pelvic injuries, optimal therapy in the face of bleeding pelvic injury is still controversial. The options for external pelvic fixation using a pelvic C clamp or an external fixator, which is called damage control orthopedics, may be considered for definitive treatment of pelvic fracture, in Europe [14]. These external pelvic interventions can effectively provide a temporary solution of the problem of hemorrhage of venous origin. However, delay in TAE for external pelvic fixation in patients with pelvic fractures would have delayed embolization in the face of ongoing arterial bleeding [15]. Reports that damage control orthopedics for multiple trauma, especially including head trauma, increased pulmonary complications, acute respiratory distress syndrome, or deterioration of outcome for multiple trauma patients have increased [16]. These complications or exacerbation of anemia due to arterial hemorrhage might increase the risk of the exacerbation of cardiac arrhythmia and cardiac function.

TAE appears to achieve its effect by controlling the arterial hemorrhage and allowing the tamponade effect of the hematoma to control venous hemorrhage [17]. Moreover, patients who undergo TAE for pelvic injury tend to be older. Kimbrell et al. suggested that an age of 60 years or older has a high likelihood of active retroperitoneal hemorrhage, regardless of the presumed hemodynamic stability on angiography, and the elderly over 60 years was

Table I. Patient's clinical data during primary trauma resuscitation

\begin{tabular}{|c|c|c|c|c|}
\hline Variables (normal) & Pre IVR & Post IVR & At 12 hours after admission & At 24 hours after admission \\
\hline Red blood cells $\left(430-550 \times 10^{4} / \mu \mathrm{l}\right)$ & 221 & 347 & 367 & 368 \\
\hline Hemoglobin $(13.5-175.5 \mathrm{~g} / \mathrm{dl})$ & 7.4 & 11.3 & 11.8 & 12.0 \\
\hline Hematocrit $(39-52 \%)$ & 22 & 32 & 34 & 34 \\
\hline PLATE $\left(15-35 \times 10^{4} / \mu \mathrm{l}\right)$ & 15.7 & 15 & 12.5 & 11.7 \\
\hline PT $(\mathrm{sec})$ & 14.9 & 14.3 & 14.0 & | $4 . \mid$ \\
\hline PT count (sec) & 12.6 & 12.6 & 12.6 & 12.6 \\
\hline APTT (sec) & 42.1 & 43.2 & 43.3 & 42.5 \\
\hline APTT count (sec) & 35.3 & 35.3 & 37.9 & 37.9 \\
\hline $\mathrm{pH}$ & 7.319 & 7.454 & 7.412 & 7.528 \\
\hline Basic deficit (mmol/l) & -2.4 & 1.9 & 0.9 & 0.5 \\
\hline
\end{tabular}

IVR, interventional radiology; PT, prothrombin time; APTT, activated partial thromboplastin time. 
the only independent predictor of the need for TAE for pelvic injury [18]. They also suggested that TAE is highly effective and less invasive in controlling hemorrhage in cases of pelvic injuries, which are difficult to manage by surgical intervention.

In the present case, surgical intervention was difficult due to his hemodynamic status. Therefore, it necessary to perform transvenous IVR for life-threatening cardiac arrhythmia immediately, and simultaneously to diagnose of arterial hemorrhage and perform TAE, if arterial hemorrhage was detected, in order to prevent metabolic and heart failure due to persistent hemorrhage were the optimal treatment options. We strongly emphasize that TAE is an effective and safe resuscitation technique for the elderly patient with secondary pelvic fractures after accidental falling due to life-threatening cardiac arrhythmia which needs interventional treatment.

\section{Abbreviations}

$\mathrm{AV}$, atrioventricular; IVR, interventional radiology; ED, emergency department; CT, computed tomography; AIS, abbreviated injury scale; SBP, systolic blood pressure; TAE, transcatheter arterial embolization; ICU, intensive care unit.

\section{Consent}

Written informed consent was obtained from the patient for publication of this case report and accompanying images. A copy of the written consent is available for review by the Editor-in-Chief of this journal.

\section{Competing interests}

The authors declare that they have no competing interests.

\section{Authors' contributions}

JM and AT were attending physicians of this patient. JM performed interventional radiology in primary trauma resuscitation and prepared the manuscript. SO did the final proof-reading of the manuscript. All authors read and approved the final manuscript.

\section{Acknowledgement}

The authors are indebted to Prof. J. Patrick Barron of the International Medical Communications Center of Tokyo Medical University for his review of this manuscript.

\section{References}

I. Talbot LA, Musio RJ, Witham EK, Metter EJ: Falls in young, middleaged and older community dwelling adults: perceived cause, environmental factors and injury. BMC Public Health 2005, 5:86.

2. Brignole M, Alboni P, Benditt DG, Bergfeldt L, Blanc JJ, Bloch Thomsen PE, van Dijk JG, Fitzpatrick A, Hohnloser S, Janousek J, Kapoor W, Kenny RA, Kulakowski P, Masotti G, Moya A, Raviele A, Sutton R, Theodorakis G, Ungar A, Wieling W; Task Force on Syncope, European Society of Cardiology:
Guidelines on management (diagnosis and treatment) of syncope - update 2004. Europace 2004, 6:467-537.

3. Martilainen K, Seppa K, Viita P, Rajala S, Laippala P, Keränen T: Transient loss of consciousness with and without injuries: when to treat these patients? Eur J Gen Pract 2003, 9:91-95.

4. Chiu T, Pang P, Ying SY, Burd A: Syncope and burns. Burns 2004, 30:438-442.

5. Omert L, Zakhary S, Wilson R, Diminno C, Rodriguez A: Falling down and falling out: management and outcome analysis. J Trauma 2004, 56:58-63.

6. Bartoletti A, Fabiani P, Adriani P, Baccetti F, Bagnoli L, Buffini G, Cappelletti C, Cecchini P, Gianni R, Lavacchi A, Ticali PF, Santoro GM: Hospital admission of patients referred to the Emergency Department for syncope. A single-hospital prospective study based on the application of the European Society of Cardiology Guidelines on Syncope. Eur Heart J 2006, 27:83-88.

7. Woolf $A D$, Akesson K: Preventing fractures in elderly people. BM] 2004, 327:89-95.

8. American College of Surgeons, Committee on Trauma: Advanced Trauma Life Support (ATLS) for Doctors; Student Course Mannual. 7th edition. Chicago: American College of Surgeons; 2004:103-115.

9. Chang-Sing P, Peter CT: Syncope: evaluation and management. Cardiol Clin I99I, 9:64|-65|.

10. Tinetti ME, Speechley M, Ginter SF: Risk factors for falls among elderly persons living in the community. $N$ Engl ] Med I988, 319:1701-I707.

II. Bartoletti A, Fabiani P, Bagnoli L, Cappelletti C, Cappellini M, Nappini G, Gianni R, Lavacchi A, Santoro GM: Physical injuries caused by a transient loss of consciousness: main clinical characteristics of patients and diagnostic contribution of carotid sinus massage. Eur Heart J 2008, 19:618-624.

12. Vardas PE, Auricchio A, Blanc JJ, Daubert JC, Drexler H, Ector H, Gasparini M, Linde C, Morgado FB, Oto A, Sutton R, Trusz-Gluza M; European Society of Cardiology; European Heart Rhythm Association: Guidelines for cardiac pacing and cardiac resynchronization therapy. The Task Force for Cardiac Pacing and Cardiac Resynchronization Therapy of the European Society of Cardiology. Developed in collaboration with the European Heart Rhythm Association. Europace 2007, 9:959-998.

13. ECC Committee, Subcommittees and Task Forces of the American Heart Association: 2005 American Heart Association Guidelines for Cardioplumonary Resuscitation and Emergency Cardiovascular Care. Circulation 2005, I I 2:IVI-203.

14. Probst C, Probst T, Gaensslen A, Krettek C, Pape HC; Study Group of the German Trauma Society: Timing and duration of the initial pelvic stabilization after multiple trauma in patients from the German Trauma Registry: Is there an influence on outcome? J Trauma 2007, 62:370-377.

15. Miller PR, Moore PS, Mansell E, Meredith JW, Chang MC: External fixation or arteriogram in bleeding pelvic fracture: initial therapy guided by markers of arterial hemorrhage. J Trauma 2003, 54:437-443.

16. Townsend RN, Lheureau T, Protetch J, Riemer B, Simon D: Timing fracture repair in patients with severe brain injuries. J Trauma 1998, 44:977-983.

17. Spahn DR, Cerny V, Coats TJ, Duranteau J, Fernandez-Mondejar E, Gordini G, Stahel PF, Hunt BJ, Komadina R, Neugebauer E, Ozier Y, Riddez L, Schultz A, Vincent JL, Rossaint R; Task Force for Advanced Bleeding Care in Trauma: Management of bleeding following major trauma: a European guideline. Crit Care 2007, I I:R I7.

18. Kimbrell BJ, Velmahos GC, Chan LS, Demetriades D: Angiographic embolization for pelvic fractures in older patients. Arch Surg 2004, 139:728-733. 